\title{
Lateral interference and perceptual grouping in visual detection
}

\author{
WILLIAM P. BANKS \\ Pomona College and Claremont Graduate School, Claremont, California \\ and \\ HEDY WHITE
Claremont Graduate School, Claremont, California
}

\begin{abstract}
The first two experiments show that recognition of target letters presented briefly in the fovea, exactly at the fixation point, is degraded by masking characters placed beside them and, further, that the amount of degradation is reduced when the masking characters are clustered into a Gestalt group with noise characters that are added to the array. Experiment 3 presents targets in the periphery at well-specified locations for essentially continuous viewing and also shows both lateral masking and the release of masking by grouping of noise characters. These results demonstrate that neither lateral masking nor the release of masking by grouping of noise can be entirely accounted for by uncertainty of location of the target. Gestalt grouping can influence the ways in which an array is searched, but, in addition, it affects the resolving power of the visual system. Implications for theories of lateral masking, feature perturbation, and Gestalt grouping effects are discussed.
\end{abstract}

When a single letter or character is seen in the company of other items, its visibility is generally reduced relative to single presentation, even when the other items need not be reported. There are a number of factors that determine the degree of interference between items in the visual field. These factors have been extensively studied in recent years in an attempt to understand the underlying mechanisms behind the interference and, in turn, to understand the mechanisms by which the visual system gathers information from the environment.

The two most important factors determining the interference among items are the distance separating them and their distance from the fovea. Identification of a single target can go from near chance to asymptotically correct performance as the distance between it and even a single nontarget is increased (Banks, Larson, \& Prinzmetal, 1979; B. A. Eriksen \& C. W. Eriksen, 1974; Krumhansl, 1977). Retinal locus affects interference in two important ways beyond the obvious decline in simple acuity from the fovea to the periphery. First, a given distance between items results in more interference as items are moved further into the periphery. To put this in a different way, the nor-

This research was supported by NIMH Grant MH 33279. The authors thank William K. Estes, William Prinzmetal, and George Wolford for their comments and advice on this research. The final version of this article was completed while the first author was on leave at the Cognitive Science Laboratory at the University of California, San Diego, under the sponsorship of Donald H. Norman. I thank Professor Norman and the rest of the staff for their generosity and the intellectual stimulation they provided. Requests for reprints should be sent to William P. Banks, Psychology Department, Pomona College, Claremont, CA 91711. $\mathrm{H}$. White is now at Psychology Department, Western Carolina University, Cullowhee, NC 28723. mal acuity gradient for a single item (i.e., the decline in visibility as the item is moved from the fovea to the periphery) is made much steeper (i.e., visibility declines more quickly) when the item has an adjacent nontarget item than when it does not (Banks, Bachrach, \& Larson, 1977; Banks, et al., 1979).

The second important influence on interitem interference related to retinal locus is the asymmetry effect. This effect is that a single nontarget item or mask placed on the peripheral side of a target reduces visibility of the target more than a single item placed on its foveal side. The asymmetry effect can be so strong that the peripheral member of a string of four items is reported more accurately than the central member (Estes, Allmeyer, \& Reder, 1976). It seems likely in many cases that some part of the difference in report accuracy comes from cognitive strategies (i.e., registering, storing in memory, and reporting the more visible items first) and only part comes from asymmetries in lateral interference at a sensory level. In fact, when non-sensory components of the task are minimized, the asymmetry effect is reduced (Banks et al., 1977, 1979).

However, it is important that the asymmetry effect is never eliminated, even in the case of continuous viewing of a display with a laterally masked target-a situation in which there seems to be no room at all for an effect of cognitive strategies. Such a case is reported in Banks et al. (1977, Experiment 3) and by Taylor and Brown (1972) and Townsend, Taylor, and Brown (1971). Similar cases can be experienced directly by the reader by examining Figure 1 or Figure 2 of Townsend et al. (1971), Figure 5 of Banks et al. (1979), or Figure 2 of this article. Woodworth $(1938$, p. 720$)$ and Woodworth and Schlosberg $(1954$, p. 104) also present figures that allow 
the reader to observe lateral masking with prolonged viewing. All of these demonstrations show lateral masking and asymmetry of masking with continuous viewing and, thus, in the absence of the cognitive strategies subjects may develop in coping with tachistoscopic presentation. The central empirical point of this article is that lateral masking, the asymmetry of masking, and the release of masking by grouping of the noise elements (discussed below) have a component not attributable to cognitive strategies such as attentional allocation or order of report. We first describe the theoretical approach that motivates this work and accounts for the results, then we discuss the alternative approaches we contrast with ours in the experiments reported here.

Our previous research on visual detection gave evidence for effects of Gestalt-like organization of the array on recognition or detection of single items in it (Banks, Bodinger, \& Illige, 1974; Banks et al., 1979; Banks \& Prinzmetal, 1976; Prinzmetal, 1980; Prinzmetal \& Banks, 1977, 1983). The general principle derived from those studies was that the greater the degree of grouping between target and noise and the larger the number of noise items with which the target is grouped, the worse the performance (Banks \& Prinzmetal, 1976; Prinzmetal \& Banks, 1977).

There are grounds for attributing at least some component of lateral masking between pairs of items in the field to the perceptual grouping between them. This is so because the principles of proximity and similarity, both venerable Gestalt laws, are also important in lateral masking and their effect is predicted by the grouping principle. That is, increasing target-noise similarity and proximity increases both the strength of grouping and the size of the lateral masking effect. Furthermore, the grouping hypothesis has an explanation for the asymmetry of lateral masking as well as for the masking effect itself. The explanation begins with the assumption that if target and noise are close enough together or similar enough to be grouped together into a single configuration, then identification of the target requires that the target's identifying features be analyzed from this configuration. Thus, when the target-plus-noise configuration is presented peripherally, it is the distance of the midpoint of this configuration from the fovea that determines performance, not the distance of the target itself from the fovea. However, in studies of the asymmetry effect reviewed by Banks et al. (1977), the typical procedure was to measure performance as a function of the eccentricity (distance from fovea) of the target and to assume that the asymmetry effect was demonstrated when placement of a noise item on the peripheral side of the target degraded performance more than did placement on the central side. The problem with this procedure is that the configuration with the peripheral mask is, as a whole, more peripheral, and hence it is in a region of less acuity than is the configuration with the central mask. Performance should therefore be worse with a peripheral mask for this reason alone. The data in Banks et al. (1977, and also those of Banks et al.,1979) support this reasoning. They showed an asymmetry effect that could be entirely accounted for by the greater overall eccentricity of the configuration with a peripheral mask. When the measure of retinal eccentricity was taken as the distance from the fixation point to the midpoint of the target-noise configuration rather than to the target alone, there was no difference in performance between peripheral and central masks and, hence, no asymmetry effect remaining after the correction for configural eccentricity.

Banks et al. (1979, Experiment 3) attempted a direct test of the configurational hypothesis. The reasoning in this experiment was that, to the extent that lateral masking is caused by grouping of target and noise, it can be released by forcing the noise element to group with elements other than the target, even though target-noise distance is kept constant. Underlying this prediction were the assumptions that an element can belong only to one group at a time and that some principle of grouping could be used to attach the noise element perceptually to elements other than the target. The principle they chose was good continuation. The new group was created by the addition of four more noise items in a column that extended two above and two below the one noise item used in the single noise configurations (the groups of noise elements in these stimuli were similar to those seen in Figures 1 and 2, below). The added configuration caused an overall improvement in performance (contrary to what might be expected from a simple display-size effect) and reversed the asymmetry effect, such that mask-peripheral conditions gave better performance than mask-central conditions. The overall improvement in performance was taken as support for the configural hypothesis because the release of masking did occur, as predicted, when the grouping of the noise item was diverted away from the target. The reversal of asymmetry was attributed (in an admittedly post hoc manner) to the increase in grouping in the periphery. When the column of noise items was peripheral to the target, it was assumed to form a stronger separate group than when it was central to it.

While we take our research as giving strong evidence for a configural component in lateral masking, we do not argue that target-noise grouping accounts for the entire interference effect. The finding of response competition effects (B. A. Eriksen \& C. W. Eriksen, 1974), for example, points to additional sources of interference. Furthermore, Chastain and Lawson (1979) found that there was still an asymmetry effect within configurations, an effect not predicted by a simple configurational hypothesis. However, the configural position does not need to assume that all of the interference is attributable to grouping processes. To support it, and the theoretical assumptions it implies, it is necessary only to show that organizational properties of the array affect the visibility of targets in ways not predictable from simpler information-processing concepts.

One account of organizational effects that attributes them to the simpler information-processing processes was recently advanced by Wolford and Chambers (1983). They proposed that the configurational properties of an array do indeed influence processing, but only by directing attention within the array. That is, they argued that the con- 
figuration of noise elements helps processing only by acting as a marker to indicate the location of the target. The effect of perceptual grouping is therefore not to influence either the confusion between target and noise features or the rate of extraction of target features, but rather only to act as a landmark in the display. Any prominent marker that served the purpose of clearly indicating the location of the target or noise would have the same result. The conclusion that grouping target and noise together causes interference or that organizing them in separate groups releases it would be rejected in favor of an attentional explanation.

The essential distinction between Wolford and Chamber's theory and ours must be spelled out very clearly. Both positions recognize that Gestalt-like grouping will influence processing, but Wolford and Chambers assert that the influence is entirely through directing attention in the array. We argue that in addition to the attentional effect of grouping, there is a preattentional effect by which features of noise and of target elements are more likely to be confused or interchanged when they fall in the same group than when they do not. Wolford and Chambers rejected such interference as an "inherent" advantage of grouping, but we think it has a natural interpretation by an extension of Wolford's (1975) own feature-perturbation model. This extension is simply that feature perturbation, which is Wolford's basic mechanism of lateral interference, is more likely within a group than between separate groups. This hypothesis has received support with paradigms somewhat different from the present target-identification paradigm. Recent work of Treisman (1982; see also Treisman \& Gelade, 1980) supports the idea that features of objects are represented perceptually in separate "maps" of the visual field, that locating targets that are defined as conjunctions of features from two of these maps requires coordination of these maps, and that the organization of the array can affect the observer's ability to find the conjunctions. Prinzmetal (1981) has shown that illusory conjunctions of features are more likely when the features fall in the same group than when they fall in different ones. If we can assume that errors in detection can come from something like an illusory conjunction between noise and target features (a "perturbation" of their features), then a preattentive effect on grouping on detection should be expected.

The general strategy of the research reported here is therefore to look for grouping effects in situations in which uncertainty of attentional allocation or of the location of the target is not a factor in detection of the target. Grouping effects found under these circumstances should not be attributable to attentional allocation and would support a preattentive influence of organizational factors. Experiments 1 and 2 present the target in the fovea at the central fixation point, and they still show a release from masking when flanking noise elements are grouped. Experiment 3 presents targets in the periphery with nontachistoscopic viewing and full information about target location, to rule out the possibility that the release of masking by grouping of noise in these experiments (as well as in Banks et al., 1979) is specific to tachistoscopic presentation. We should add that Experiments 1 and 2 were conducted prior to our acquaintance with the research reported in Wolford and Chambers (1983), and that neither these nor Experiment 3 were designed as a reply to that work. However, Wolford and Chambers put forward a position that very cogently states the general position to which this research was addressed, namely that the Gestalt effects can be completely reduced to the allocation of attention.

\section{EXPERIMENTS 1 AND 2}

These experiments present all targets at the fixation point while varying the configuration of flanking masks. They differ only in that eye movements are monitored in Experiment 2 but not in Experiment 1. Both experiments use an attention-dividing task to control accuracy. Pilot work showed that it was very difficult to avoid ceiling effects with foveal presentation. After 100 trials, our pilot subjects approached asymptotic performance with the shortest stimulus duration our system could present. We therefore introduced a concurrent task and found that it could be used to keep performance far from $100 \%$. The concurrent task required subjects to judge whether two single digits presented in the periphery, placed symmetrically on either side of the central target, had the same or different "oddity." That is, subjects made one response if both digits were odd or both were even, and made the other response if one was odd and the other was even.

The initial reason for using this particular concurrent task was not simply to reduce accuracy but to maximize the chance of discovering a grouping effect if it existed. The fovea provides a uniquely well-specified location in the visual field, but it is also unfortunately the area with the weakest tendency to form perceptual groups (Beck, 1972), possibly because attention is habitually focused there. Distributing attention with a concurrent task has been shown to increase grouping effects and would be likely to maximize the probability of discovering a grouping effect in the fovea if it exists. In any event, if we had manipulated the visual aspects of the display to degrade performance to an equivalent degree, we would have risked destruction of configural effects by degrading the configuration itself.

\section{Method}

\section{Experiment 1}

Subjects. The subjects were 14 male and female undergraduates from the Claremont Colleges, who were either paid $\$ 8$ or received course credit for the approximately $2 \mathrm{~h}$ of the experiment. They all had 20/30 (corrected) vision, or better, and they were allowed to wear glasses or contact lenses during experimental trials.

Procedure. Subjects began by reading typed instructions explaining both the numerical "oddity" task (described above) and the detection task. In the detection task, the target always appeared exactly at the fixation point, and the subject's task was to decide whether a $T$ or an $F$ had been presented. The target letter was presented briefly along with the masking letters (Hs), whose presentation was coterminous with the target. After their initial questions were answered, the subjects were given four practice trials (with 50 -msec presentation of both oddity and detec- 
tion stimuli) to acquaint them with the stimuli. They then had two blocks of 20 stimuli each for practice, a short rest period in which they could ask questions or discuss the task, and then the experimental trials. The experimental trials had 40 blocks of 20 stimuli each (for a total of 800 responses), with brief rests after each set of 5 blocks and a longer rest (30 min) after the 20th block.

In an experimental block, the first trial was initiated by the subject's keypress. Immediately after the keypress, a fixation point appeared on the screen for $250 \mathrm{msec}$. Then the pair of numbers appeared simultaneously with the stimulus letter and masking material, all of which stayed on the screen for $17 \mathrm{msec}$. The target letter always appeared at the fixation point, and was replaced by the fixation point at the end of the $17 \mathrm{msec}$. The subject used a hand-held box containing two microswitches to be used in responding. The subject responded first to the oddity task and second to the letter-detection task. After the second response, the computer presented feedback about each of the two tasks separately by presenting "correct" or "incorrect" for $500 \mathrm{msec}$ for each of the tasks. The first feedback word reported on performance on the numerical task and the second on the detection task. After the second feedback stimulus, the fixation point came on for $250 \mathrm{msec}$ and the sequence of events began again. The computer put the detection stimuli in random order before each block and generated random pairs of digits for the oddity task on each trial. The detection response was discarded on trials in which the subject gave the wrong response on the oddity task, and the detection stimulus from that trial was repeated at the end of the block in which the error had occurred.

Stimuli and Design. All stimuli were presented on a 10-in. General Electric Model 10AB0402V color television driven (in black and white) by an Apple II+ microcomputer. All letters were in the Apple uppercase format. Target luminance was approximately $21 \mathrm{fL}$ against a background of $1.8 \mathrm{fL}$. The subject sat in an adjustable chair with a firm, somewhat constraining headrest of the sort supplied with dental chairs. The chair was individually adjusted for an eye-screen distance of $1.0 \mathrm{~m}$ for each subject.

All detection stimuli had a $\mathrm{T}$ or an $\mathrm{F}$ centered on the fixation point and either no mask, a single masking letter (always on $\mathrm{H}$ ), or a column of $\mathrm{Hs}$ on either the left or the right side. Figure 1 depicts a target with one mask and with the column of grouped masks. The letters and numbers were all presented within a space $.29^{\circ}$ wide and $.34^{\circ}$ high. The distance between the target and the nearest noise letter was $.57^{\circ}$ centerto-center or approximately $.28^{\circ}$ edge-to-edge. The Hs in the column were spaced with their centers separated by $.23^{\circ}$. Their nearest edges were separated by approximately $.06^{\circ}$. The digits were always placed symmetrically, one on the left and one on the right, along the horizontal line that contained the fixation point, target, and nearest $H$. The center of each digit was $3.25^{\circ}$ from the fixation point.

The 20 stimuli in each block represented two presentations of each of the 10 orthogonal combinations of the variables in the detection task. The 10 combinations result from the two targets' ( $F$ or $T$ ) being presented under the five mask conditions: single $\mathrm{H}$ on the left or right side, column of Hs on left or right, or no mask at all. Half of the 20 stimuli had number pairs requiring a left microswitch response, and half had pairs with a right response. The specific numbers satisfying these constraints were selected by a random process before each trial.

\section{Experiment 2}

As mentioned, the only important difference between Experiments 1 and 2 was the monitoring of eye movements. Because the oddity task

$\begin{array}{rr}H & \\ H & \\ \text { TH } & 5 \\ H & \\ H & \end{array}$

Figure 1. Depiction of stimulus array used in Experiments 1, 2, and 3, printed approximately to scale. In this example, the masking characters (Hs) are in the grouped configuration, the target is T, and the peripheral numbers for the concurrent task are 5 and 6. draws attention to the periphery, it was considered likely that on at least some trials eye movements would occur and effectively place the target in the periphery. Therefore, it seemed necessary to replicate Experiment 1 and discard trials on which an eye movement had occurred. Because of the difficulty of monitoring eye movements, fewer subjects and fewer trials were used, but more practice was given.

Subjects. The subjects were five students from the Claremont Colleges. Three of the students volunteered in partial fulfillment of their duties as research assistants and the other two subjects were each paid $\$ 8$ for the approximately $2 \mathrm{~h}$ required by the experiment.

Procedure. After five blocks of practice, the subjects received 20 blocks (400 trials) of experimental trials. Eye movements were monitored in both practice and experimental trials by means of a closed-circuit television system. This system used a camera with a macro lens placed about 8 in. from the subject's left eye. The image of the eye was approximately 6 in. wide on a 20 -in. diagonal TV monitor, with the cornea having a diameter in the image of approximately 2 in. An experimenter in the room with the subject observed the eye on the monitor to detect eye movements. This system was adopted because it is more sensitive, more reliable, and more flexible than any of the other nonintrusive monitoring systems that were available to us. The experimenter could easily detect $.25^{\circ}$ of movement, and the system was not subject to problems such as shifting calibration that plagued the corneal reflection system we had used in the past (Banks et al., 1977, 1979).

When the subject made the final response on a trial, the computer waited for the experimenter's response before starting the next trial. The experimenter had two response keys: one signaled the computer to treat the trial as an error trial; the other signaled the computer to accept the trial. Either response caused it to proceed to the next trial. The experimenter could not see the experimental stimuli but knew a trial was initiated because the computer was programmed to emit the carriage return tone at the onset of each trial, and the sound of the subject's responses on the microswitch signaled the end of the trial to the experimenter. The experimenter considered the occurrence of any eye movement during the interval from the tone to the first microswitch closure to invalidate the trial, even though the stimulus itself occupied only $17 \mathrm{msec}$ of this period.

Practice was intended in part to give subjects feedback about eye movements to help them learn to keep their eyes still during data trials. Further training in holding eyes fixated seemed unnecessary. The subjects had virtually no trouble maintaining fixation from the first trial, and very few trials were discarded because of eye movements. Practice, with variations in the difficulty of the oddity task, was also intended to allow calibration of detection accuracy. The oddity task was made more difficult by moving the numbers further into the periphery. If accuracy on a practice or experimental block was over $75 \%$ ( 15 out of 20 ), the digits were moved further into the periphery; if it was under $75 \%$, they were moved toward the fovea. The mean distance of the digits from the fovea varied from $2.9^{\circ}$ to $4.3^{\circ}$ over subjects.

\section{Results and Discussion}

Both experiments showed both masking and a release of masking with the grouping of masking items. In Experiment 1 , proportion correct for no mask (single target only) was .894 , and this dropped to .838 for a single mask but rose to .882 for grouped mask. In Experiment 2, accuracy was .912 with no mask, dropped to .845 for a single mask, and rose to .886 for grouped masks. Given that chance performance was .50 in both experiments, the release of masking corresponds to $8.8 \%$ of the total range in Experiment 1 and $8.2 \%$ in Experiment 2; to put it another way, grouping the noise eliminated $79 \%$ of the masking effect in Experiment 1 and $61 \%$ of the masking in Experiment 2.

The main effect of noise condition was reliable for both Experiment 1 and Experiment $2[\mathrm{~F}(4,52)=12.35$, 
$p<.01$ and $F(4,16)=3.03, p<.05$, respectively]. The simple masking effect (contrast between no mask and single mask) was reliable in both experiments $[F(1,52)=20.9$ for Experiment 1 and $F(1,16)=8.06$ for Experiment 2, both ps < .02]. In Experiment 1, the contrast between grouped and single noise was reliable $[\mathrm{F}(1,52)=12.9$, $\mathrm{p}<.01]$, but in Experiment 2, with fewer subjects, it fell short of reliability $[F(1,16)=3.02, .10>p>.05]$.

In Experiment 1, but not Experiment 2, there was a reliable interaction between grouping and mask position (left vs. right side of target) $[\mathrm{F}(1,52)=5.23, \mathrm{p}<.05]$. For grouped masks, accuracy was $88.1 \%$ for left and $88.4 \%$ for right placement, but for single masks it was $82.0 \%$ when the mask was on the left and $85.6 \%$ when it was on the right. The better performance with grouped than with single masks was reliable in both cases $[F(1,52)=$ $27.31, \mathrm{p}<.01$, for displays with masks on the left and $F(1,52)=4.23, p<.01$, for displays with masks on the right].

Both experiments showed an increase in accuracy over blocks. In Experiment 1, mean accuracy rose from 78.0\% on the first half to $89.4 \%$ on the second half [for blocks, $F(7,91)=12.35, p<.01]$ and from $82 \%$ to $93 \%$ in Experiment $2[\mathrm{~F}(1,4)=7.26, .05<\mathrm{p}<.06]$. In Experiment 1 , there were no interactions with practice, but in Experiment 2, the grouping effect was stronger in the second half of practice than in the first $[F(4,16)=3.09$, $\mathrm{p}<.05]$.

Several analyses were performed to determine whether there was an interaction or interference between the oddity task and the letter-detection task. Because the two experiments gave similar results but Experiment 2 had many fewer data, the analyses were not performed with the second experiment. First, an analysis of the RTs to the oddity task showed no effects of the masking condition in which they fell, but the "same" response was faster than the "different" response $[1.70 \mathrm{vs.} 1.98 \mathrm{sec}$ with $\mathrm{F}(1,77)$ $=12.9, \mathrm{p}<.01]$. The RT for the oddity judgment was correlated negatively with accuracy on the detection task over the 10 major conditions of the experiment $(r=$ -0.42 ). Accuracy on the oddity judgment correlated positively with accuracy on the detection task $(r=+.19)$. These correlations suggest that some interference may exist between the two tasks, but they are difficult to interpret. One possibility (supported by other data reported below) is that subjects made both choices before responding. A difficult detection task could therefore slow the response to the oddity task, and vice versa, even though the choices were made independently.

We looked in two ways for interference caused by the common response mode used by the two tasks. First, response key (left vs. right) for the oddity task was used as a factor in the original analysis of detection accuracy. Only one effect involving key approached reliability. This was an interaction between the response key used on the two tasks such that detection responses using the same key as the oddity task on the trial were $3.5 \%$ less accurate than those using a different key $[F(1,13)=3.49, p<.10]$. Since response key for the oddity task was orthogonal with this (and all other) detection factors, this effect does not contaminate any of the effects of interest.

Second, we analyzed detection RTs to look for further evidence for competition between the two tasks as well as for a possible speed-accuracy tradeoff in the detections. Since these responses occurred after the oddity task responses, it is difficult to know whether to measure the RTs from the offset of the stimulus or from the completion of the oddity response. Planning for the second response could take place before the first response, during the interval between the two, or during some combination of both. The data suggest that the detection choice was largely made prior to the response on the oddity task because the detection response followed it by a mean of only $133 \mathrm{msec}$. This would be an extremely fast mean RT if it measured the whole time for the decision, particularly for a decision seemingly as difficult as this one. Our main analysis of variance was therefore on the RT measured from the offset of the display. This analysis showed no reliable effects of any of the variables in the detection experiment and no evidence of conflict between the two responses. The speed-accuracy relationship was measured by correlations between detection accuracy and RT over the 10 main conditions of the experiment, measured both from the offset of the display and from the execution of the oddity response. The former correlation was -.45 and the latter, -.18 . With neither measure of detection RT was there any evidence for a speed-accuracy tradeoff.

Experiment 1 shows that with the target position well marked and at the fovea, there is still a reliable effect of the configuration of flanking noise elements. The fact that foveal targets are subject to lateral masking and that grouping of noise elements releases the masking implies that release of masking by grouping of noise does not result solely from reduction of positional uncertainty. Experiment 2 replicated the results of Experiment 1 with eye movements controlled. This replication shows that the results of Experiment 1 are not created by subjects' moving their eyes away from the foveal fixation point and thereby placing the target and masking letters in the periphery. We note that even though overt eye movements did not take place during experimental trials, covert shifts of attentional focus could have taken place. Of course, such shifts could not affect the retinal position of the targets, but they could create a situation of subjective uncertainty about target position. This uncertainty could create a configural effect attentionally. We argue in response, first, that such covert shifts of attention would be as nonoptimal for performance as overt eye movements (both would increase the ease of encoding one digit at the expense of the other). We would therefore want good evidence for them. Second, Experiment 3, in which attentional focus is not an issue, leads to the same conclusions.

\section{EXPERIMENT 3}

This experiment uses a nontachistoscopic, effectively unlimited viewing paradigm to test for lateral masking, the asymmetry of masking, and the release of masking by 
grouping of noise. There are several reasons to test for these effects nontachistoscopically. Lateral interference seems more definitely a perceptual effect and less likely a result of cognitive strategies when demonstrated with unlimited rather than with tachistoscopic viewing. In particular, attentional effects or strategies such as those proposed by Wolford and Chambers to account for the grouping effect apply to tachistoscopic presentations in which subjects can use the positional cue given by the group to optimize their scanning of a briefly presented array. They are not plausible when stimuli are available for long durations of exposure in which subjects can freely vary their attentional focus over the array. Thus, if a release of masking with grouping is still obtained with nontachistoscopic presentation, the attentional hypothesis would not be supported. An additional reason to test for grouping effects nontachistoscopically is to provide evidence to converge with that of Experiments 1 and 2. Those experiments reduced positional uncertainty to the vanishing point in one way; this one reduces it in a different way. If both lead to the same conclusions, our confidence in the inference is increased.

This experiment was conducted in a manner very similar to that of Experiments 2 and 3 of Banks et al. (1977). In those experiments targets with either peripheral or central masks were moved slowly in steps toward the fovea from the periphery, and the degree of eccentricity was noted at which the target was seen. Those experiments demonstrated asymmetry of masking but did not investigate possible configural effects (which, in fact, were not proposed as an explanation of asymmetry before Banks et al., 1979). In this experiment the mask was on either the central or the peripheral side of the target and, in addition, it was either a single mask or a mask grouped by good continuation like those in Experiments 1 and 2. The grouping variable allowed a test of whether grouping improves performance in nontachistoscopic viewing and whether it can release (or reverse; cf. Banks et al., 1979) the asymmetry effect.

\section{Method}

Subjects. Five subjects, two female and three male, ranging in age from 19 to 40 years and all with 20/20 uncorrected vision or better, served for two separate sessions of approximately $1 \mathrm{~h}$ each. The subjects were individually fitted with biteboards molded to the shape of the upper jaw, and they used these boards in all sessions. Three of the subjects were paid, one served in partial fulfillment of his duties as a research assistant, and one was the first author.

Apparatus. The apparatus used in Experiments 2 and 3 of Banks et al. (1977) was modified for the present research. This apparatus consisted of a large, $1.2 \mathrm{~m}$ high $\times 1.6 \mathrm{~m}$ wide, black panel with a central $53 \mathrm{~cm}$ high $\times 75 \mathrm{~cm}$ wide flap that was lifted to expose the stimulus array in a $25 \mathrm{~cm}$ high $\times 60 \mathrm{~cm}$ wide window. A piece of white posterboard was placed in the window to provide a backdrop for the stimuli. The stimuli were Stenso Gothic rub-on letters on strips of blank, white, $19 \mathrm{~cm}$ high $\times 7.5 \mathrm{~cm}$ wide cardstock. These strips were hung in the window over the top edge of the posterboard in such a way that the subjects could see them from the front and the experimenter standing behind the posterboard could move them.

On each trial, the experimenter selected a stimulus card and hung it on the top of the posterboard so that the stimulus area of the card was facing the subject. The experimenter then opened the flap to expose the stimulus and moved it toward the fixation point until the subject was able to identify the target letter.

The subject viewed the stimuli while the biteboard kept his or her eyes $3.0 \mathrm{~m}$ from the screen. The subject fixated a cross on either the left or the right of the stimulus screen, and held this fixation as the stimuli were moved from the periphery toward the center of the visual field. On each trial, the subject was told in advance whether the target was placed on the peripheral or central side of the noise.

Eye movements were monitored by the same closed-circuit video display that was used in Experiment 2. Two experimenters were required: one to control the stimuli and record data and one to watch the TV monitor and report any eye movement during an experimental trial.

Stimuli and Design. All cards contained one of the three targets (uppercase A, Y, or T) and either one mask or a column of grouped masks on the left or right side. There were two mask and target separations, termed "near" and "far." The mask was a nonletter figure made by combining either Es or Hs from the letter set with horizontal or vertical bars to make a "hybrid E-H" that looked like a pair of vertically aligned, contiguous squares. Figure 2 shows sample stimuli from the near and far conditions for both single and grouped masks. The stimuli shown in the four cells of Figure 2 are photographic copies of stimuli used in the experiment. The orthogonal combination of three targets, left vs. right placement of the mask, near vs. far separation, and grouped vs. single mask, created 24 stimuli. In addition, the set of 24 was augmented by 9 more stimuli, representing three copies of each of the three targets on cards with no mask at all. The experimental set thus had a total of 33 stimuli.

The stimuli were evenly front-illuminated by a mixture of incandescent and fluorescent light. The cardstock and posterboard both had the same luminance, $9.4 \mathrm{fL}$; the black strokes of the rub-on letters had a luminance of $.8 \mathrm{fL}$. Stroke width for all solid lines was $.57^{\circ}$. Letters and masks fell within a rectangle $.113^{\circ}$ wide $\times .25^{\circ}$ high. The center-tocenter spacing of items was $.15^{\circ}$ in the near condition and $.26^{\circ}$ in the far condition. The target and the adjacent noise items were thus nearly touching at their closest points (separated by $.037^{\circ}$ ) in the near condition. The noise element was $.113^{\circ}$ wide $\times .25^{\circ}$ high. The grouped configuration of noise elements was $1.36^{\circ}$ high, and the vertically aligned noise items were separated by $.033^{\circ}$ from edge to edge or $.28^{\circ}$ center to center.

Procedure. Each session was devoted to testing a single visual field. Three of the subjects had the right field tested first, and two had the

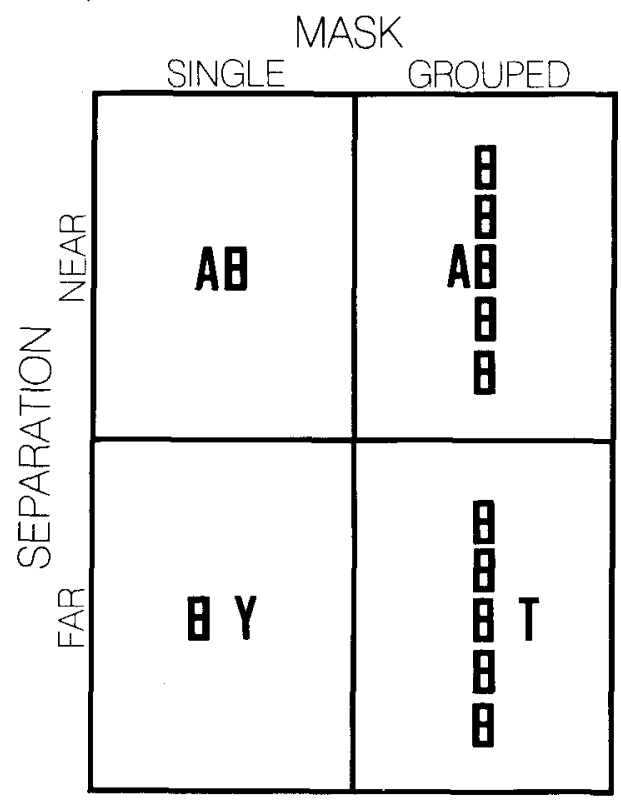

Figure 2. Examples of the four categories of stimuli used in Experiment 3. These are photographic reproductions of the stimulus cards. 
left. The same apparatus was used for both visual fields, but the subjects fixated a cross on the left of the display for testing of the right visual field and a cross on the right for testing of the left visual field.

A session began with the subject adjusting the biteboard and chair for maximum comfort. The video camera was adjusted and calibrated by having the subject shift fixation back and forth between selected points in the visual display. The experimenter adjusted the camera until the eye movements could be detected. In most cases, eye movements smaller than $.25^{\circ}$ could be detected, but such precision was probably unnecessary. The type of eye movement that caused data trials to be aborted was almost always a saccade that darted to the target position, usually in the range of $2^{\circ} 5^{\circ}$ away.

Before data trials were begun, the subject had from 10 to 20 trials of practice to become accustomed to the task and to learn to suppress eye movements. (Three of the subjects quickly learned to suppress eye movements. The other two did not easily learn to suppress them and lost about $25 \%$ of their trials because of eye movements.) Once practice trials were over, the subjects were tested on all 33 stimuli in a single block with frequent ad-lib rest breaks. The order of the 33 stimuli was randomized with the use of a random number table before each session.

On a given trial, the subject assumed fixation, and the experimenter behind the screen placed a stimulus card at the extreme peripheral end of the display, at approximately $10^{\circ}$ from the fixation point. This location was much too peripheral to allow any subjects to detect any of the stimuli there (the mean point of detection was $3.31^{\circ}$ ). The eccentricity at which the target was seen was determined by a procedure similar to a method of limits with ascending trials only. After the flap was raised to expose the stimuli, the stimulus card was moved toward the fixation point in $.5^{\circ}$ steps. The card was left to rest for $2 \mathrm{sec}$ at each step and then moved $.5^{\circ}$ closer and left to rest again for 2 sec. This process was repeated until the subject identified the stimulus. The 2 -sec duration was chosen because pilot work had shown that exposure durations beyond $2 \mathrm{sec}$ added little to accuracy of identification; $2 \mathrm{sec}$ thus seemed a practical approximation to continuous viewing. All subjects were instructed not to guess but rather to report the target only when they could see it clearly. If a subject made an incorrect identification, he or she was reminded to wait until the target was clearly visible, and the stimulus card that led to the error was shuffled into the deck and presented later. Errors were not recorded by stimulus, but the total tally showed that all subjects had fewer than $10 \%$ errors.

\section{Results and Discussion}

The nontargets in general greatly degraded visibility of the targets. Single targets were seen at a mean distance of $4.8^{\circ}$ from fixation, whereas the mean over all arrays with one or more noise elements was $2.75^{\circ}[\mathrm{t}(4)=5.9$, $\mathrm{p}<.01]$. Although they had strong effects on visibility of the targets, the variables of greatest interest, grouped versus single noise elements and central versus peripheral placement of the mask, had their effects almost entirely in two interactions, grouping $\times$ spacing and grouping $\times$ asymmetry. These two interactions are shown in Figures 3 and 4. Figure 3 shows the interaction between grouping and target-mask spacing. When target and mask are close together, the grouped mask gives worse visibility than the single mask, and the reverse is true for the wider spacing $[F(1,4)=8.17, p<.05]$. This result replicates the finding of Wolford and Chambers (1983) with tachistoscopic presentations.

The interaction between grouping and peripheral versus central placement of the mask is shown in Figure 4. This result replicates, with $\mathrm{F}(1,4)=11.5, \mathrm{p}<.05$, the finding of Banks et al. (1979) that a grouped mask reduces the asymmetry effect. This pattern was essentially the same for both target-mask distances, and the three-way interac-
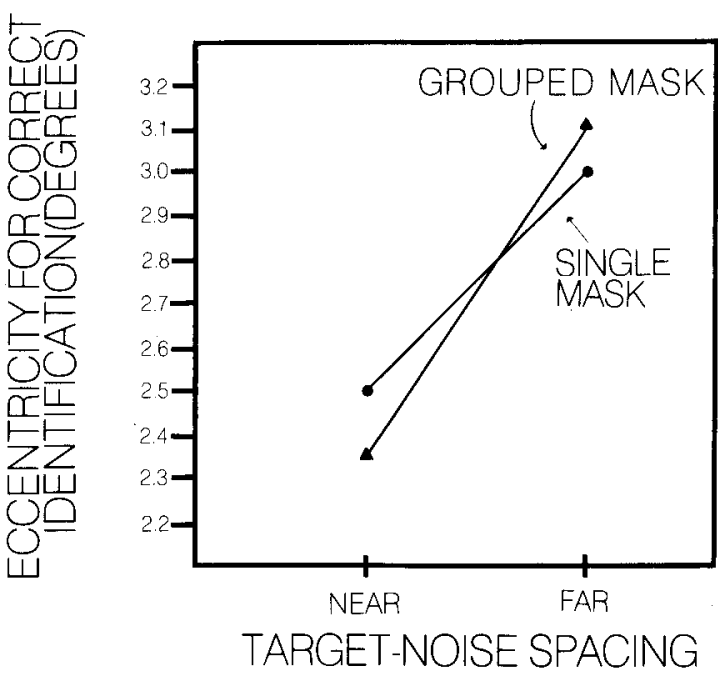

Figure 3. Mean eccentricity of target identification in Experiment 3 for grouped and single noise items and near and far target-noise spacing.

tion of grouping, mask placement, and target-mask distance had an $F<1.0$.

The only reliable main effects were target-noise distance $[F(1,4)=54.2, p<.002]$ and target identity $[F(2,8)=$ $10.1, \mathrm{p}<.01]$. The distance between target and noise has frequently been shown to be an important variable in lateral masking, and previous research with letters from this type font has shown the same pattern of differences among the target letters, namely that the target $Y$ gives considerably worse performance than the other targets (Banks et al., 1977). As in the previous research, none of the principal conclusions depends on the identity of the target.

The overall asymmetry effect fell short of reliability $[F(1,4)=2.52, .20>p>.10]$. This overall weak effect seems to derive from the conditions with grouped masks, which had very small asymmetry effects (only $.14^{\circ}$; cf. Figure 4) and reduced the main effect of asymmetry.

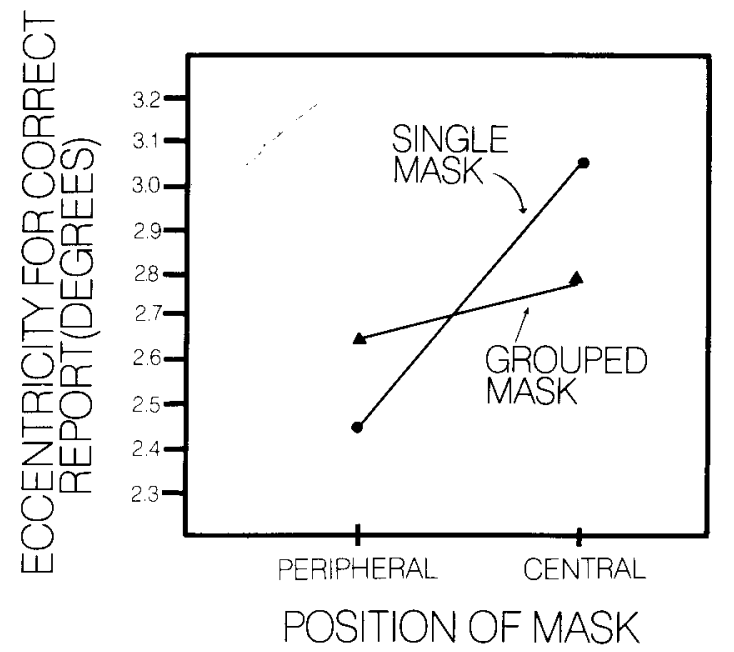

Figure 4. Mean eccentricity of target identification for grouped and single noise items and peripheral and central masks in Experiment 3. 
When only the single-mask condition is considered, the asymmetry effect is larger $\left(.61^{\circ}\right)$ and is reliable $[F(1,4)=$ 4.7, $\mathrm{p}<.05]$. A similar result was obtained in Experiment 3 of Banks et al. (1979), in which a reliable asymmetry effect in the expected direction was observed with a single mask but not with a grouped mask. The situation there was actually somewhat more extreme than in the present case, because, with the grouped mask, the asymmetry effect reversed, showing reliably better performance with a peripheral mask than with a central one. Here the asymmetry effect declined sharply when the mask was grouped, but it did not reverse.

The overall grouping effect was insignificant $(F<1.0)$, and even went very slightly $\left(.02^{\circ}\right)$ in the unexpected direction. In Figure 3, it is seen that the reason for the overall lack of a main effect of grouping lies in the choice of targetmask spacing. If shorter target-mask spacings had been used, we would have found an overall decline in performance with grouping of noise elements. With larger spacings, we would expect the more typical improvement in performance with noise grouping. Thus, the overall lack of a grouping effect seems to have come from the choice of target-noise spacing.

The interaction beween grouping and spacing replicates Wolford and Chambers's (1983) result, but the experimental conditions are so different that we do not think that the explanation they gave can apply. Their explanation attributed the advantage of grouping to the way in which the group provides a marker for the target and thus reduces uncertainty about target position. In this experiment, however, uncertainty about target position is entirely removed. During experimental trials, although subjects found it easy to attend to the target and noise positions in their periphery, they still had difficulty identifying the target. (The reader may be able to experience this difficulty by peripheral viewing of the arrays in Figure 2.) It seems best to consider other explanations of the grouping effect than positional uncertainty.

The explanation advanced by Banks et al. (1979) for tachistoscopic presentations also applies here. This is that grouping the noise prevents it from grouping with the target. Lateral masking is reduced by separate grouping of target and noise because featural transposition or perturbations are less likely between groups than within groups. This explanation holds for the far spacing, but why does the effect reverse for near spacing? In this case, the near spacing was approximately the same as the spacing between the grouped noise elements. Common interelement spacing is a strong grouping principle (Wertheimer, 1958). As can be seen in Figure 2, the target (A) in near spacing seems to group with the grouped noise elements perhaps even more strongly than with the single noise element. It seems likely, then, that the target simply joined in the larger group when more noise items were added. ${ }^{1}$

The interaction between grouping and asymmetry (Figure 3) has no explanation under the attentional allocation hypothesis, but can be explained by considerations concerning grouping. The attentional hypothesis does predict the improvement in performance with grouping a peripheral mask. The problem, however, comes with the decline in performance when a central mask is grouped, because it should act equally well as a marker or cue in either case. The attentional allocation hypothesis necessarily predicts, contrary to the data, an improvement when a mask on either side of the target is grouped. The configural hypothesis, on the other hand, predicts just this pattern of results (as it predicted a similar one in Banks et al., 1979). Grouping the peripheral noise element has the effect of forming a configuration that tends to exclude the target, releasing it from some of the interference from the noise elements. Grouping the central noise is not as likely to form a separate configuration, because the tendency of items to group declines toward the fovea. When the noise items are not grouped together, the target is seen among a larger group of noise items and performance is worse because of the added noise items.

\section{GENERAL DISCUSSION}

The results of the four experiments in this study show that the effects of noise-elements grouping do not depend on the group's acting as a marker to direct the subject's attention. We discuss below two relatively straightforward ways in which the grouping effect could depend on attentional allocation, showing how they are at odds with at least several features of our results. We do not assert that attentional effects are entirely absent from the various configural effects reported in the literature, but the present results show that configural effects are still found when attention is already optimally directed and configurationdirected shifts cannot improve performance. We conclude this section with a version of the positional uncertainty hypothesis that seems to be consistent with our own configural effects as well as with Wolford's and Treisman's findings.

The two attentional accounts of grouping effects attribute them either (1) to the ease of finding the target in the visual field (the entire target-plus-noise cluster is easier to locate when the noise is grouped) or (2) to allocation of attention over the elements in the array (there is less uncertainty about which element should be processed first when the noise is grouped than when it is not).

The problem for the first account is clear in all three experiments. When the target is always presented exactly at the fixation point (as in Experiments 1 and 2) or when it is presented for a long time at a well-specified location (Experiment 3), there seems to be little room for uncertainty about where to find it in the field, and the attentional explanation is not plausible. Furthermore, this account does not predict the result found in Experiment 3 (or in Banks et al., 1979), in which a grouped mask on the foveal side of the target reduces visibility of the target. Because visibility is improved when the noise is grouped on the peripheral side of the target (see Figure 4), it should also be improved when the noise is grouped on the foveal side. The interaction seen in Figure 4 is therefore quite damaging to this hypothesis, and we can think of no way to repair it that is not blatantly ad hoc. The 
hypothesis that the grouping effect in these experiments results from the ease of locating the target with a grouped configuration is therefore incompatible with our results.

The second hypothesis, that grouping of noise facilitates appropriate allocation of attention over the array, is plausible for the tachistoscopic presentation of peripheral stimuli. In tachistoscopic presentation, the subject must make an efficient allocation of effort in the brief time the stimulus is available, and locating the target within a peripherally presented array can be difficult. However, in Experiments 1 and 2, the target was foveally presented and its location was quite well specified. The hypothesis is much less plausible in such a case, although it is not completely implausible. In Experiment 3, however, the mode of presentation did not restrict the available energy or the amount of time needed to process it, and the subject was always told whether the target was on the foveal or the peripheral side of the array. Thus, the subjects knew exactly where the target was located both absolutely and relatively in the array. They did not need to make a quick guess about which was the target element in a rapidly fading trace, but could apply their attention steadily to the region containing the target information. The fact that grouping of noise elements could still release the interference and reduce the asymmetry effect under these conditions implies that the effect of grouping does not depend on the form of the array giving direction to cognitive strategies. In fact, cognitive strategies would seem to play a very small role under the conditions of Experiment 3. Effects found under these conditions should therefore largely reflect influences of array configuration on the resolving power of the visual system.

Another piece of evidence relevant to the question of whether configural effects depend on allocation of attention is contained in Prinzmetal (1980). That study used arrays like those in Banks and Prinzmetal (1976). When subjects judged whether an $\mathrm{F}$ or a $\mathrm{T}$ was presented in one of these arrays, they were faster and more accurate when the noise was grouped than when the entire array was a good form. The question Prinzmetal (1980) addressed was whether the configuration of noise elements in the grouped noise condition facilitated processing by directing attention to the target or whether grouping had an effect over and above the attentional one. To answer this question, Prinzmetal presented both kinds of array with a cue that was an arrow that pointed at the position of the target in the array. The cue came on simultaneously with the array or 10,50 , or $100 \mathrm{msec}$ before it. (A control condition had a noninformative cue that simply signaled the onset of the array.) The results show that the informative cue greatly facilitated processing, and the configural effect was highly reliable and almost perfectly additive with cuing, showing an $\mathrm{F}<1.0$ for the interaction between cue interval and noise grouping. Thus, although attentional effects were quite strong, the purely configurational effect was additive with them and therefore independent of them. This result is similar to that of Colegate, Hoffman, and C. W. Eriksen (1973), who found that the display-size effect was nearly constant over the range of SOA used. In both cases, it seems reasonable to conclude that the limits on performance imposed by display variables (array configuration or number of elements) are not the same limits as those that are reduced by the cue.

Wolford and Chambers's (1983) interpretation of configural effects is that the configuration acts as a simultaneous cue (SOA $=0$ ) to the location of the target. We do not dispute that some component of the measured configural effects reported in the literature may derive from such cuing, but the available evidence indicates that the attentional direction given by perceptional grouping and the isolation between noise and target features it allows are separate components of the configurational effect that operates by different processes. This evidence includes the present research and previous research (Banks et al., 1979, Experiment 3) in which a configural effect was found when there was no uncertainty about target location for the configuration to reduce. We also cite the Banks and Prinzmetal (1976) article in which the configuration had no "cue validity" (to use the term of Wolford \& Chambers, 1983). That is, in the Banks and Prinzmetal study, the configuration of grouped noise elements was not a reliable cue to the location of the target in the array. When the noise was grouped, it could still appear in any one of the four possible target locations; three of these were within the noise with which subjective boundary-drawing showed it to be grouped, and only one was outside of the noise. The "cue validity" of the configurations in these studies was therefore weighted toward directing subjects to search within the configuration, yet performance was much better on trials in which the target was separate from the group ( $25 \%$ of the time) than when the target was in the group (75\% of the time). In this case, the preattentive configural effect was apparently stronger than the cuing effect provided by the array.

Wolford and Chambers (1983, Experiment 4), on the other hand, reported a result that we interpret as showing that strategic effects can sometimes be stronger than the preattentive grouping effect. They tachistoscopically presented arrays similar to those used in the present Experiments $1-3$, but the target was $5.23^{\circ}$ to the left or right of fixation. They had three groups of subjects: One had conditions similar to those of the present and previous experiments with a single target and grouped versus single noise items (Banks et al., 1977, 1979), and this condition replicated the advantage of grouping the noise. A second condition reversed the target-noise assignment such that, in the grouped arrays, a column of grouped targets was in the target location and the noise item was on either the central or peripheral side of it. This condition gave even better performance than the first one. The third condition had a mixture of the two types of grouped array, and performance was worse than in either of the other conditions. Wolford and Chambers argue that the poor performance in the mixed condition results from the destruction of the cue validity of the configuration. However, performance in the mixed condition is not consistent with a purely attentional model, ${ }^{2}$ and we suggest that the apparently unusual demands of this condition elicited cog- 
nitive strategies that are not normally used and that obscured whatever configural effects that may have existed in other conditions. We think that a more direct and easily interpreted experimental strategy of comparing cuing and configural structure would be a better attack on the problem.

In sum, our evidence shows that grouping can affect detection performance independently of its possible role in directing attention. We propose that perceptual organization operates by increasing perturbations of target and noise features within groups and reducing them between groups. This process in letter identification would seem to be the same as that studied for illusory conjunctions by Prinzmetal (1981). It is reasonable to conclude that a laterally masked letter is one that has suffered illusory conjunctions. Attention seems to have two roles: In addition to the obvious one of directing order of readout from the array, it can also impose an organization on the array when it is not preattentively organized (or is inappropriately organized) for the task by the configural structure. Banks and Prinzmetal (1976) and Kahneman (1973) have referred to this as "reparsing," an attentive process assumed to change the organization of an array (as, for example, when we can "will" a tile floor to look like rows, columns, diagonals, and so forth). One possible role of this process would be to change the preattentive figureground organization to reduce featural intrusions on target locations in an array. Another would be to establish the locations of features in an array to strengthen the normal grouping process that bonds features into a single object. The latter role would be illustrated by Treisman and Schmidt's (1982) experiment in which featural confusions among objects occurred only when attention was divided.

We conclude that featural confusions in letter detection arise from the same process as illusory conjunctions, and are subject to the same effect. More confusions occur within than between Gestalt clusters of items, whether they are letters or shapes, and attention can, to some degree, overcome the effects of preattentive Gestalt grouping. Although a portion of Gestalt effects (particularly with tachistopic presentation) may be attributable to strategies of array inspection that utilize the easily visible configurations in the array, this research shows that configural structure also affects the visibility of items in ways that do not depend on allocation of attention, even in continuously viewed arrays.

\section{REFERENCES}

Banks, W. P., Bachrach, K. M., \& Larson, D. W. (1977). The asymmetry of lateral interference in visual letter identification. Perception \& Psychophysics, 22, 232-240.

Banks, W. P., Bodinger, D., \& Illige, M. (1974). Visual detection accuracy and target-noise proximity. Bulletin of the Psychonomic Society, 2, 411-414.

Banks, W. P., Larson, D. W., \& Prinzmetal, W. (1979). Asymmetry of visual interference. Perception \& Psychophysics, 25, 447-456.

Banks, W. P., \& PrInzmetal, W. (1976). Configurational effects in visual information processing. Perception \& Psychophysics, 19, 361-367.
BECK, J. (1972). Similarity grouping and peripheral discriminability under uncertainty. American Journal of Psychology, 85, 1-19.

Chastain, G., \& LaWson, L. (1979). Identification asymmetry of parafoveal stimulus pairs. Perception \& Psychophysics, 26, 363-368.

Colegate, R. L., Hoffman, J. E., \& ERIKSEn, C. W. (1973). Selective encoding from multielement visual displays. Perception \& Psychophysics, 14, 217-224.

ERIKSEN, B. A., \& ERIKSEN, C. W. (1974). Effects of noise letters upon the identification of a target letter in a nonsearch task. Perception \& Psychophysics, 16, 143-149.

Estes, W. K., Allmeyer, D. H., \& Reder, S. M. (1976). Serial position functions for letter identification at brief and extended exposure durations. Perception \& Psychophysics, 19, 1-15.

KaHneman, D. (1973). Attention and effect. Englewood Cliffs, NJ: Prentice-Hall.

KrUmhansL, C. L. (1977). Naming and locating simultaneously and sequentially presented letters. Perception \& Psychophysics, 22, 293-302. 293-302.

Prinzmetal, W. (1980). Simultaneous and successive presentation in target detection and search tasks. Unpublished doctoral dissertation, Claremont Graduate School.

Prinzmetal, W. (1981). Principles of feature integration in visual perception. Perception \& Psychophysics, 30, 330-340.

Prinzmetal, W., \& Banks, W. P. (1977). Good continuation affects visual detection. Perception \& Psychophysics, 21, 389-395.

Prinzmetal, W., \& Banks, W. P. (1983). Perceptual capacity limits in visual detection and search. Bulletin of the Psychonomic Society, 21, 263-266.

TAYLOR, S. G., \& BRowN, D. R. (1972). Lateral visual masking: Supraretinal effects when viewing linear arrays with unlimited viewing time. Perception \& Psychophysics, 12, 97-99.

Townsend, J. T., TAYLOR, S. G., \& BROWN, D. R. (1971). Lateral masking for letters with unlimited viewing time. Perception \& Psychophysics, 10, 375-378.

Treisman, A. M. (1982). Perceptual grouping and attention in visual search for features and for objects. Journal of Experimental Psychology: Human Perception and Performance, 8, 194-214.

Treisman, A. M., \& Gelade, G. (1980). A feature-integraton theory of attention. Cognitive Psychology, 12, 97-136.

Treisman, A., \& Schmidt, H. (1982). Illusory conjunctions in the perception of objects. Cognitive Psychology, 14, 107-141.

Werthermer, M. (1958). Principles of perceptual organization. In M. Wertheimer \& D. C. Beardslee (Eds.), Readings in perception. New York: D. Van Nostrand.

Wolford, G. (1975). Perturbation model for letter identification. Psychological Review, 82, 184-199.

Wolford, G., \& Chambers, L. (1983). Lateral masking as a function of spacing. Perception \& Psychophysics, 33, 129-138.

WoODWORTH, R. S. (1938). Experimental psychology. New York: Holt. WOODWORTH, R. S., \& SCHLOSBERG, H. (1954). Experimental psychology. New York: Holt, Rinehart, and Winston.

\section{NOTES}

1. We attempted to assess the grouping structure of the array by having subjects give direct judgments of structure by drawing boundaries in Xerox copies of the arrays. Previous research (Banks \& Prinzmetal, 1976; Prinzmetal \& Banks, 1977) had shown that subjective organization of arrays predicted detection performance, although Wolford and Chambers (1983) had not. In this assessment, four of our subjects (excluding William P. Banks), after their second session, were given copies of all 24 stimuli containing noise elements and were asked to draw boundaries around groups or "blobs" as they had appeared during experimental trials. The results showed only an effect of target-noise separation: For the far separation, the sum over all variables gave 94 out of a total of 96 boundaries falling between target and noise; for the near separation, it was 2 out of 98 falling between target and noise. The subjective reports of grouping in the array were thus dominated by target-noise separation, which was also the strongest single variable in the detection experiment. This result supports our contention that spacing affects grouping, and 
thus that the decline in performance with close spacing could result from target-noise grouping. However, the drawn boundaries do not show any difference between grouped and nongrouped noise. It seems possible that target-noise distance created such a strong organization of the array that the subjects did not notice, or did not report, the other aspects of array organization. It is also possible that the presentation at the fovea and with a larger visual angle than that in the experiment altered the grouping structure. Organizational effects of good continuation are as well documented as those of proximity, and we do not suppose that they were absent here. It would have been better to have been able to report a quantitative measure of the effect of good continuation on subjective organization in our arrays, but it seems unlikely that the principle did not operate on this case.

2. Accuracy of target detection in Wolford and Chambers's (1983) Experiment 4 with grouping in the display was $54.4 \%$ in mixed trials, but approximately $69 \%$ for the condition with multiple grouped targets and $58 \%$ for those with multiple grouped masks (the $54.4 \%$ is computed from information given in Wolford \& Chambers; the latter two percentages are estimated from their Figure 4). The attentional allocation hypothesis attributes the decrement in the mixed condition to the lack of cue validity of the configuration: On any given trial, the target will be unpredictably found in the group or alone. In such a situation, the cue validity hypothesis would have different predictions, depending upon what strategy subjects adopt. If they always inspected the same area of the array (grouped column or single item), behaving, in effect, as though they were in one of the other two conditions, then they would perform at a level halfway between that condition and chance. This is so because on the $50 \%$ of trials when target does fall at the location they monitor, they will perform at the level pertaining to the target location they monitor (58\% if they monitor the location separate from the group and $69 \%$ if they monitor the group); on the remaining $50 \%$ of trials they would perform at chance. The observed accuracy of $54.4 \%$ is quite close to the $54 \%$ that would be predicted if subjects monitored the single item and too small to correspond to the prediction of approximately $59.5 \%$ if they had monitored the grouped items. It would seem, then, that they monitor the item separate from the group, but this hypothesis predicts that they would perform at chance on trials with grouped targets. This prediction fails because they actually did slightly better when the target was grouped than when the noise items were grouped ( $54.8 \%$ vs. $54 \%$ ). The alternative hypothesis, that subjects unsystematically chose or alternated between the two target locations, is at odds with two aspects of the data. First, the overall proportion correct is too low to allow more than a very small probability of monitoring the group, and, second, the proportion correct on the trials with a grouped target is much higher than would be expected if the position away from the group were the one monitored.

We should temper our ciritcisms of these results by noting, first, that many of the inconsistencies are close to the empirical resolution of the experiment (the smallest reliable differences in this experiment seem to be around $3 \%$ ) and, second, that more complex hypotheses of attentional strategies could probably account for the results. Nevertheless, these inconsistencies suggest that the mixed condition may induce subjective strategies that obscure whatever configural effects exist. Although the mixed condition of Wolford and Chambers represents an ingenious attack on the cue-validity question, the inconsistencies in the results argue that some other experimental approach, such as that of cuing locations in grouped versus ungrouped arrays, should be used to analyze the extent to which the configural effect is preattentive.

(Manuscript received April 26, 1984; revision accepted for publication July 19, 1984.) 